Mikail N ${ }^{* 1}$; Louedec L ${ }^{1}$; Aid $\mathrm{R}^{2}$; Al Shoukr F² Anizan $\mathrm{N}^{2}$ Aerts $\mathrm{J}^{2}$; Michel JB ${ }^{2}$; Chauvierre $\mathrm{C}^{2}$; Le Guludec $\mathrm{D}^{1}$; Rouzet $\mathrm{F}^{1}$

${ }^{1}$ Médecine Nucléaire, Hôpital Bichat, Paris

${ }^{3} \mathrm{U} 1148$, Inserm, Paris

nidaa.mikail@gmail.com (Mikail)

\title{
Détection de la mémoire ischémique myocardique par le 99mTc-fucoïdane : validation pré-clinique
}

Objectifs : L'identification rétrospective d'un syndrome coronarien aigu résolu pose un problème diagnostique. Certaines molécules d'adhésion, telles que les sélectines, persistent à la surface de l'endothélium après la fin de l'ischémie. Elles représentent de ce fait une empreinte de cet épisode dans ce qui est communément appelé la mémoire ischémique. Le but de cette étude est d'évaluer l'utilité du fucoïdane radiomarqué au 99mTechnetium, un agent ciblant la P- et la E-sélectine, dans la détection rétrospective d'épisodes d'ischémiereperfusion sur un modèle de rat. Matériels et Méthodes : Des épisodes transitoires d'ischémie myocardique ont été générés par ligature coronaire chez des rats mâles Wistar. La durée de l'ischémie variait entre 5 et 20 min et le délai après reperfusion entre 2 et 24 heures. Les acquisitions TEMP/TDM ont été réalisées 2 heures après injection de $70 \mathrm{MBq}$ de $99 \mathrm{mTc}$ fucoïdane. L'analyse scintigraphique a consisté en la détermination visuelle de l'existence d'une fixation focale myocardique et en une analyse semi-quantitative par calcul du rapport de fixation du cœur sur le compartiment sanguin (RCS). Les animaux ont par la suite été sacrifiés et leur cœur explanté, congelé et sectionné pour analyse autoradiographique (quantification par calcul du rapport entre la région myocardique à risque et une région myocardique saine) et histologique/immunohistologique. Résultats : Pour un délai constant de 2 heures après reperfusion, une fixation myocardique de $99 \mathrm{mTc}$-fucoïdane a été observée pour des durées d'ischémie de 20 minutes (8/8 rats, RCS : 2,4 +/- 0,9 [2,0-4,7]), 10 minutes (10/10 rats, RCS : 3,6 +/- 1,7 [1,5-7,5]) et 5 minutes (8/12 rats, RCS : 2,6 +/- 1,8 [1,9-7,1]). Sur l'autoradiographie, le rapport de fixation entre les zones à risque et saines était de 4,5 +/$1,9[3,2-9 ., 3]$ à $20 \mathrm{~min}, 7,4+/-3,1[2,8-12,1]$ à $10 \mathrm{~min}$ et $5,6+/-2,1[3,4-8,6]$ à $5 \mathrm{~min}$. Les régions fixant intensément le $99 \mathrm{mTc}$-fucoïdane coïncidaient topographiquement avec 1'expression endothéliale de P-sélectine. Après 6 heures de reperfusion, la fixation de $99 \mathrm{mTc}$ fucoïdane était détectée après 20 minutes d'ischémie (7/7, RCS : 3,0 +/- 0,6 [2,8-4,5]), 10 min d'ischémie (8/8 rats, RCS : 2,9 Conclusions : Cette étude démontre que des épisodes d'occlusion coronaire brefs suivis de reperfusion de durées variables, allant jusqu'à 24 heures, sont associés à l'expression endothéliale de P- et E-sélectine, détectables in vivo par la TEMP/TDM au 99mTc-fucoïdane. Ces données soutiennent la possibilité d'une utilisation clinique du 99mTc-fucoïdane dans la détection de la mémoire ischémique.

Les auteurs ne déclarent aucun conflit d'intérêt.

Mots Clefs : Cardiologie, Perfusion myocardique, Infarctus, TEMP/TDM, Scintigraphie 